\title{
Synergistic antitumoral effect of vinblastine and HSV-Tk/GCV gene therapy mediated by albumin-associated cationic liposomes
}

\author{
H. Faneca ${ }^{a}$, A. Faustino ${ }^{\text {b }}$, M.C. Pedroso de Lima ${ }^{a, b, *}$ \\ ${ }^{a}$ Center for Neuroscience and Cell Biology, University of Coimbra, 3000 Coimbra, Portugal \\ ${ }^{\mathrm{b}}$ Department of Biochemistry, Faculty of Science and Technology, University of Coimbra, 3000 Coimbra, Portugal
}

Received 12 September 2007; accepted 3 December 2007

Available online 14 December 2007

\begin{abstract}
Despite recent advances in conventional therapeutic approaches for cancer, the frequently observed acquired drug resistance and toxic side effects have limited their clinical application. The main goal of this work was to investigate the combined antitumoral effect of vinblastine with HSV-Tk/GCV "suicide" gene therapy mediated by human serum albumin (HSA)-associated lipoplexes, in mammary adenocarcinoma cells (TSA cells). Our results show that, among the different lipoplex formulations tested, HSA-associated complexes prepared from EPOPC:Chol liposomes, at the $(4 / 1)(+/-)$ charge ratio, was the most efficient to mediate gene delivery, even in the presence of serum. The simultaneous addition of vinblastine and HSA-EPOPC:Chol/DNA (+/-) (4/1) lipoplexes to TSA cells improved transgene expression more than 10 times. When combined with the HSV-Tk/GCV "suicide" gene therapy mediated by HSA-EPOPC:Chol/DNA (+/-) (4/1) lipoplexes, vinblastine induced a great enhancement in the antitumoral activity in TSA cells. Most importantly, this combined strategy resulted in a significant synergistic effect, thus allowing the use of a much lower dose of the drug to achieve the same therapeutic effect. Overall, our results indicate that this approach has the potential to overcome some major limitations of conventional chemotherapy, and may therefore constitute a promising strategy for future applications in antitumoral therapy.
\end{abstract}

(C) 2007 Elsevier B.V. All rights reserved.

Keywords: Cancer gene therapy; "Suicide" gene therapy; Vinblastine; Gene delivery; Cationic liposomes; Transfection

\section{Introduction}

One of the major challenges in the application of gene therapy as a therapeutic tool is to overcome the inefficient delivery of therapeutic genes into target cells. To this regard, many efforts have been made to develop efficient, safe and stable gene delivery systems [1-4]. In the process of gene delivery, many cellular obstacles have to be overcome to achieve satisfactory levels of transfection activity: binding of lipoplexes to the cell surface; uptake of the lipoplexes, endocytosis being the main pathway; escape of lipoplexes from the endolysosomal compartment and translocation through the nuclear envelope [5-9]. In this context, different strategies have

\footnotetext{
* Corresponding author. Center for Neuroscience and Cell Biology, University of Coimbra, 3000 Coimbra, Portugal. Tel.: +351 239820 190; fax: +351 239 853607.

E-mail address: mdelima@ci.uc.pt (M.C. Pedroso de Lima).
}

been developed to surpass these barriers aiming at enhancing transgene expression. For instance, in accordance with our previous observations, the association of HSA to lipoplexes seems to be a promising strategy resulting in an enhancement of transgene expression in different types of cells, even in the presence of serum $[10,11]$. This increase of tranfection efficiency most likely results from the promotion of lipoplex internalization via endocytosis and escape from the endocytotic pathway.

In the last years, gene therapy has emerged as a promising strategy for cancer treatment [12-14]. The "suicide" gene approach is a cancer gene therapy strategy that consists of the transfer into cancer cells of a "suicide" gene, which encodes the enzyme responsible for the conversion of an inactive drug into a toxic metabolite that causes cell death $[15,16]$. Herpes Simplex Virus thymidine kinase gene (HSV-tk) is one of the most promising "suicide" genes, ganciclovir (GCV) being its respective prodrug [17-19]. Cells expressing the HSV-tk gene 
metabolize GCV to ganciclovir monophosphate, which is further converted into ganciclovir triphosphate by cellular kinases. As the resulting compound is an analogue of deoxyguanosine triphosphate, inhibiton of DNA polymerase and/or incorporation into DNA will occur, leading to chain termination and tumoral cell death [17-19]. A crucial element of HSV-Tk/GCV gene therapy is the "bystander effect", by which a high percentage of tumoral cell death can occur even when only a low percentage of cells have been transfected [10,20-22].

Vinblastine is a chemotherapeutic drug that belongs to the class of microtubule-depolymerizing agents and binds specifically to tubulin, inhibiting its polymerization and the subsequent association of microtubules. As the microtubules are crucial for the occurrence of chromosomal segregation during anaphase in mitosis, the resulting depolymerization of this cytoskeleton element results in a G2-M cell cycle arrest and consequently in cell death [23]. However, microtubules are not only involved in the chromosomal segregation, but also play important roles in intracellular dynamics, including the transport of lipoplexes to lysosomes after their uptake through endocytosis [24-26]. The compounds that affect the depolymerization state of microtubules may be used to enhance the transfection capacity of lipoplexes, by helping to overcome the endolysosomal entrapment, and consequently DNA degradation at the lysosomal level [24-26]. Moreover, it has been reported that these agents can increase transfection efficiency of cationic liposome/DNA complexes by activating the sequencespecific transcription factor NF-kB [23,24,27].

Therefore, our hypothesis is that vinblastine could act in concert with HSV-Tk/GCV "suicide" gene therapy, by increasing lipoplex biological activity and inhibiting mitosis, thus leading to a synergistic antitumoral effect. In this context, the main goal of this work was to evaluate the effect of this drug on transgene expression mediated by cationic liposomes and the antitumoral activity of a combined strategy involving association of vinblastine and HSV-Tk/GCV "suicide" gene therapy mediated by HSA-associated lipoplexes, in mouse mammary adenocarcinoma cells in culture.

\section{Material and methods}

\subsection{Cells}

TSA cells (BALB/c female mouse mammary adenocarcinoma cell line) were maintained at $37^{\circ} \mathrm{C}$, under $5 \% \mathrm{CO}_{2}$, in Dulbecco's modified Eagle's medium-high glucose (DMEM-HG) (Irvine Scientific, Santa Ana, CA) supplemented with $10 \%$ (v/v) heatinactivated fetal bovine serum (FBS) (Sigma, St. Louis, MO), penicillin $(100 \mathrm{U} / \mathrm{ml})$ and streptomycin $(100 \mu \mathrm{g} / \mathrm{ml})$. TSA cells grow in monolayer and were detached by treatment with a trypsin solution $(0.25 \%)$ (Sigma, St. Louis, MO).

\subsection{Preparation of cationic liposomes and their complexes with DNA}

Small unilamellar cationic liposomes (SUV) were prepared by extrusion of multilamellar liposomes (MLV) composed of
1:1 (mol ratio) mixtures of (1) 1,2-dioleoyl-3-(trimethylammonium) propane (DOTAP) and cholesterol (Chol), (2) 1palmitoyl-2-oleoyl-sn-glycero-3-ethylphosphocholine (EPOPC) and dioleoylphosphatidylethanolamine (DOPE), or (3) EPOPC and Chol; and composed of a 2:1:1 (mol ratio) mixture of (4) EPOPC, DOPE and Chol. EPOPC:Chol (1:1) liposomes were labeled with $1 \%$ rhodamine-dioleoylphosphatidylethanolamine (rhodamine-PE), for binding and uptake studies, and with $0.1 \%$ carboxyfluorescein-dioleoylphosphatidylethanolamine (carboxyfluorescein-PE), for intracellular distribution studies. Briefly, lipids (Avanti Polar Lipids, Alabaster, $\mathrm{AL}$ ) dissolved in $\mathrm{CHCl}_{3}$ were mixed at the desired molar ratio and dried under vacuum in a rotatory evaporator. The dried lipid films were hydrated with deionized water to a final lipid concentration of $6 \mathrm{mM}$ and the resulting MLV were then sonicated for $3 \mathrm{~min}$ and extruded 21 times through two stacked polycarbonate filters of $50 \mathrm{~nm}$ pore diameter using a Liposofast device (Avestin, Toronto, Canada). The resulting liposomes (SUV) were then diluted five times with deionized water and filter-sterilized utilizing $0.22 \mu \mathrm{m}$ pore-diameter filters (Schleicher \& Schuell, Dassel, Germany). The suspension was stored at $4{ }^{\circ} \mathrm{C}$ until use. Lipoplexes were prepared by sequentially mixing $100 \mu \mathrm{l}$ of a HEPES-buffered saline solution (HBS) (100 mM NaCl, $20 \mathrm{mM}$ HEPES, $\mathrm{pH}$ 7.4), with liposomes (the volume necessary to obtain the desired (+/-) lipid/DNA charge ratio) and with $100 \mu \mathrm{l}$ of HBS solution containing $1 \mu \mathrm{g}$ of pCMVluc encoding luciferase (a gift of Dr. P. Felgner, Vical, San Diego, CA), $1 \mu \mathrm{g}$ of pCMVtk encoding HSV-tk (a gift of Prof. N. Düzgünes, University of Pacific, San Francisco, USA), or $1 \mu \mathrm{g}$ of pCMVgfp, encoding green fluorescent protein (GFP) (Clontech, Mountain View, CA). The mixtures were further incubated for $15 \mathrm{~min}$ at room temperature. For lipoplexes containing HSA (Sigma, St. Louis, MO), liposomes were preincubated with this protein ( $32 \mu \mathrm{g} \mathrm{HSA} / \mu \mathrm{g}$ of DNA) for $15 \mathrm{~min}$, followed by a further $15 \mathrm{~min}$ incubation with plasmid DNA solution at room temperature. Lipoplexes were used immediately after being prepared.

\subsection{Transfection activity}

For transfection studies with complexes containing the plasmids pCMVluc or pCMVtk, $0.4 \times 10^{5}$ TSA cells were seeded in $1 \mathrm{ml}$ of medium in 48-well culture plates $24 \mathrm{~h}$ before transfection, in order to obtain $50-70 \%$ confluence. Cells were covered with $0.3 \mathrm{ml}$ of DMEM-HG (without serum, unless indicated otherwise) before lipoplexes (containing $1 \mu \mathrm{g}$ of DNA) were gently added in a volume of $0.2 \mathrm{ml}$ per well, without or with different amounts of Vinblastine (depending on the desired concentration). After $4 \mathrm{~h}$ incubation $\left(5 \% \mathrm{CO}_{2}\right.$ at $37^{\circ} \mathrm{C}$ ) the medium was replaced with DMEM-HG and the cells were further incubated for $48 \mathrm{~h}$. Cells were then washed twice with phosphate-buffered saline solution (PBS) and $150 \mu \mathrm{l}$ of lysis buffer (1 mM DTT; $1 \mathrm{mM}$ EDTA; $25 \mathrm{mM}$ Tris-phosphate $(\mathrm{pH}=7.8) ; 8 \mathrm{mM} \mathrm{MgCl}_{2} ; 15 \%$ glycerol; $1 \%(\mathrm{v} / \mathrm{v})$ Triton X$100)$ were added to each well. The level of gene expression in the lysates was evaluated by measuring light production by luciferase in a LMax II 384 luminometer (Molecular Devices, 
Union City, CA). The protein content of the lysates was measured by the Dc Protein Assay reagent (Bio-Rad, Hercules, CA) using bovine serum albumin as the standard. The data were expressed as RLU of luciferase per mg of total cell protein.

For transfection studies with complexes containing the plasmid pCMVgfp, $1.2 \times 10^{5}$ TSA cells were seeded in $2 \mathrm{ml}$ of medium in 12-well culture plates (previously covered with a coverslip), $24 \mathrm{~h}$ before transfection. Cells were covered with $0.5 \mathrm{ml}$ of DMEM-HG before lipoplexes (containing $2 \mu \mathrm{g}$ of DNA) were added in the presence or absence of $0.5 \mu \mathrm{M}$ vinblastine. Following $48 \mathrm{~h}$ after the initial transfection period $(4 \mathrm{~h})$, cells were fixed with $4 \%$ paraformaldehyde, for $15 \mathrm{~min}$ at room temperature, the nuclei were labeled with the fluorescent DNA-binding dye Hoechst $33342(1 \mu \mathrm{g} / \mathrm{ml})$, for $5 \mathrm{~min}$ at room temperature, and then cells were mounted in Vecta Shield mounting medium (Vecta Laboratories Inc., Burlingame, CA, USA). Images were taken using a confocal microscope (LSM-510 META, Zeiss), under the 63x oil immersion objective.

\subsection{Cell viability assay}

Following transfection under the different experimental conditions, cell viability was assessed by a modified Alamar Blue assay [28]. The assay measures the redox capacity of the cells due to the production of metabolites as a result of cell growth and allows determination of viability over the period culture without the detachment of adherent cells. Briefly, $0.4 \mathrm{ml}$ of $10 \%$ (V/V) Alamar Blue dye in complete DMEMHG medium was added to each well $45 \mathrm{~h}$ following the initial transfection period $(4 \mathrm{~h})$. After $1 \mathrm{~h}$ of incubation at $37^{\circ} \mathrm{C}, 200 \mu \mathrm{l}$ of the supernatant were collected from each well and transferred to 96-well plates. The absorbance at 570 and $600 \mathrm{~nm}$ was measured in a SPECTRAmax PLUS 384 spectrophotometer (Molecular Devices, Union City, CA). Cell viability (as a percentage of control cells) was calculated according to the formula $\left(A_{570}-A_{600}\right)$ of treated cells $\times 100 /\left(A_{570}-A_{600}\right)$ of control cells.

\subsection{Antitumoral activity}

The in vitro antitumoral activity mediated by non-viral HSV-Tk/GCV gene therapy, vinblastine or their combination was evaluated in TSA cells. Since the production of ganciclovir was discontinued, its prodrug valganciclovir (VGCV), an L-valyl ester of GCV, was used. Following $4 \mathrm{~h}$ incubation of TSA cells with HSA-associated lipoplexes or HBS, in the presence or absence of $0.5 \mu \mathrm{M}$ vinblastine, the medium was replaced with normal DMEM-HG. Twenty-four hours after, the medium was replaced with DMEM-HG with or without different concentrations of VGCV $(100,200$ or $400 \mu \mathrm{M})$ and cells were further incubated for 5 days under cultured conditions $\left(5 \% \mathrm{CO}_{2}\right.$ at $\left.37^{\circ} \mathrm{C}\right)$. The medium, with or without VGCV, was replaced daily and the cell viability was assessed every other day by the modified Alamar Blue assay as referred above.

\subsection{Binding and uptake of lipoplexes}

EPOPC:Chol (1:1) liposomes, labeled with 1\% rhodamine$\mathrm{PE}$, were complexed in the presence or absence of HSA, with $1 \mu \mathrm{g}$ of pCMVluc at a $4 / 1(+/-)$ cationic lipid/DNA charge ratio. Twenty-four hours before transfection, $0.5 \times 10^{5} \mathrm{TSA}$ cells were seeded in $1 \mathrm{ml}$ of medium in 48-well culture plates. After covering the cells with $0.3 \mathrm{ml}$ of DMEM-HG medium, lipoplexes were added in the presence or absence of $0.5 \mu \mathrm{M}$ vinblastine. Following a $2 \mathrm{~h}$ incubation at $4{ }^{\circ} \mathrm{C}$ or $37^{\circ} \mathrm{C}$, cells were washed twice with PBS and lysed with $100 \mu 1 /$ well of $1 \%$ Triton X-100. Binding $\left(4{ }^{\circ} \mathrm{C}\right)$ and uptake $\left(37^{\circ} \mathrm{C}\right)$ of lipoplexes were monitored in a SPECTRAmax GEMINI EM fluorometer (Molecular Devices, Union City, CA) by measuring the fluorescence at excitation and emission wavelengths of 545 and $587 \mathrm{~nm}$, respectively.

\subsection{Intracellular distribution of lipoplexes}

Twenty-four hours before transfection, $1.2 \times 10^{5}$ TSA cells were added to each well of a 12-well culture plate, previously covered with a coverslip.

To observe the intracellular distribution of the complexes, EPOPC:Chol liposomes labelled with $0.1 \%$ carboxyfluorescein-PE and associated to HSA, were complexed with $2 \mu \mathrm{g}$ of pCMVluc at the (4/1) (+/-) charge ratio. Cells were incubated, during $30 \mathrm{~min}$, with $200 \mathrm{nM}$ LysoTracker Red DND-99 (Molecular Probes), which labels acidic compartments of live cells, and then covered with $0.5 \mathrm{ml}$ of DMEM-HG medium before lipoplexes were added in the presence or absence of $0.5 \mu \mathrm{M}$ vinblastine. After $2 \mathrm{~h}$ of incubation, the nuclei were labeled with the fluorescent DNA-binding dye Hoechst 33342 $(1 \mu \mathrm{g} / \mathrm{ml})$, for $5 \mathrm{~min}$ at room temperature, and cells were mounted in Vecta Shield mounting medium (Vecta Laboratories Inc., Burlingame, CA, USA). Images were taken with a confocal microscope (LSM-510 META, Zeiss), under the 63x oil immersion objective.

\subsection{Statistical analysis}

Data were analysed using the Prism software (version 4.0). Statistical significance of differences between data was evaluated by One-way ANOVA using the Tukey test. A value of $p<0.05$ was considered significant.

\section{Results}

\subsection{Biological activity and cytotoxicity of the complexes}

There are several parameters that affect the final physicochemical properties of lipoplexes and consequently the levels of transfection mediated by these non-viral gene carriers [1-4]. The nature of the cationic and helper lipids and the relative proportion of cationic lipid and DNA are examples of two crucial parameters that should be considered in the preparation of lipoplexes. In the present work, cationic lipid/DNA complexes with various lipid compositions and prepared at different 
charge ratios $(+/-)$ were evaluated in terms of their biological activity in TSA cells.

Fig. 1A shows that the biological activity mediated by lipoplexes in TSA cells was dependent on their charge ratio $(+/-)$ and lipid composition. Among the different formulations tested, EPOPC:Chol/DNA and DOTAP:Chol/DNA complexes were those exhibiting the highest levels of transfection $(p<0.001$ and $p<0.01$, respectively). Since the biological activity of the complexes could be affected by their cytotoxicity, cell viability studies were performed for the tested formulations. The results presented in Fig. 1B reveal that the viability of TSA cells was not significantly affected $(p>0.05)$ upon incubation with the complexes, similar results being observed for the different formulations.

Our previous results demonstrated that association of albumin to lipoplexes is highly effective in enhancing transfection
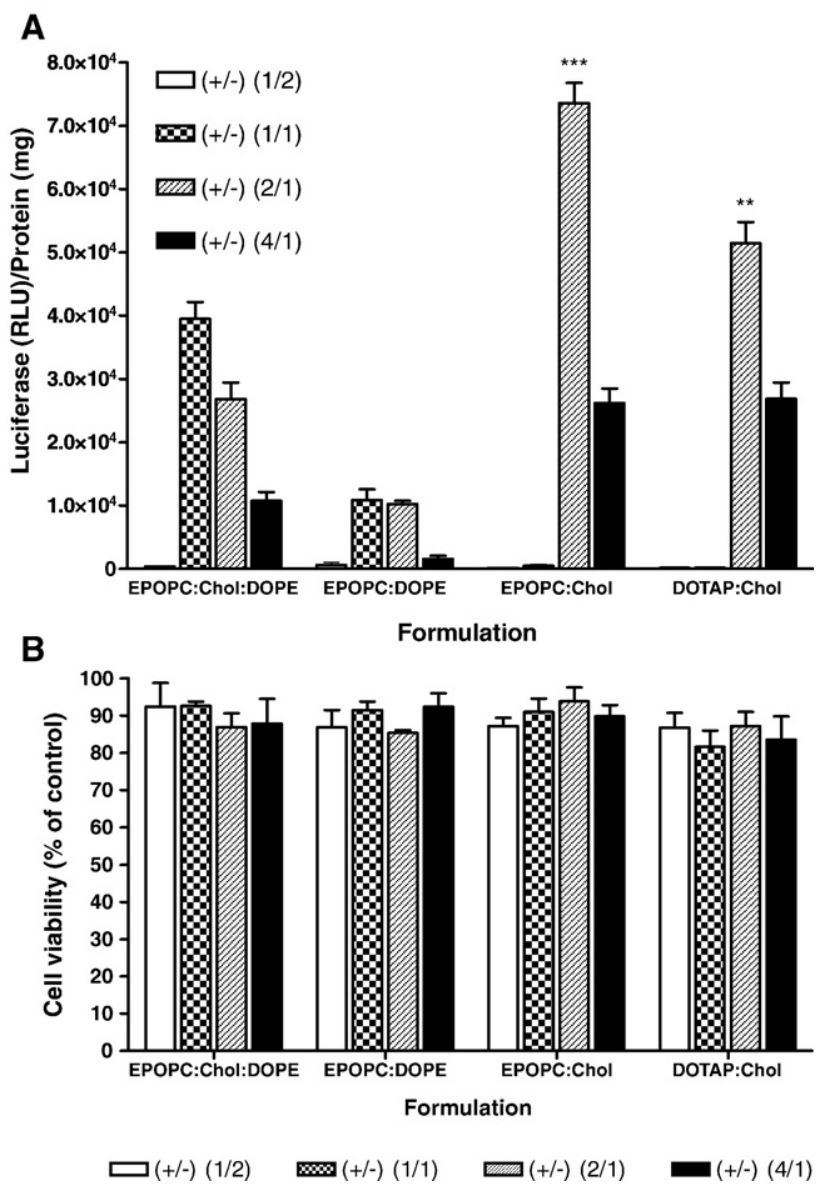

Fig. 1. Effect of lipoplex composition and charge ratio (+/-) on luciferase gene expression (A) and viability (B) in TSA cells. Liposomes with different lipid composition were complexed with pCMVluc at the indicated $(+/-)$ charge ratios. (A) Levels of luciferase gene expression are presented as RLU of luciferase per $\mathrm{mg}$ of total cell protein (mean \pm S.D. obtained from triplicates), and are representative of at least three independent experiments. Asterisks $\left({ }^{* *} p<0.01,{ }^{* * *} p<0.001\right)$, for the two best conditions, indicate values that significantly differ from those measured for the other conditions. (B) Values of cell viability measured by the Alamar Blue assay are expressed as the percentage of the untreated control cells (mean \pm standard deviation obtained from triplicates) and are representative of at least three independent experiments.

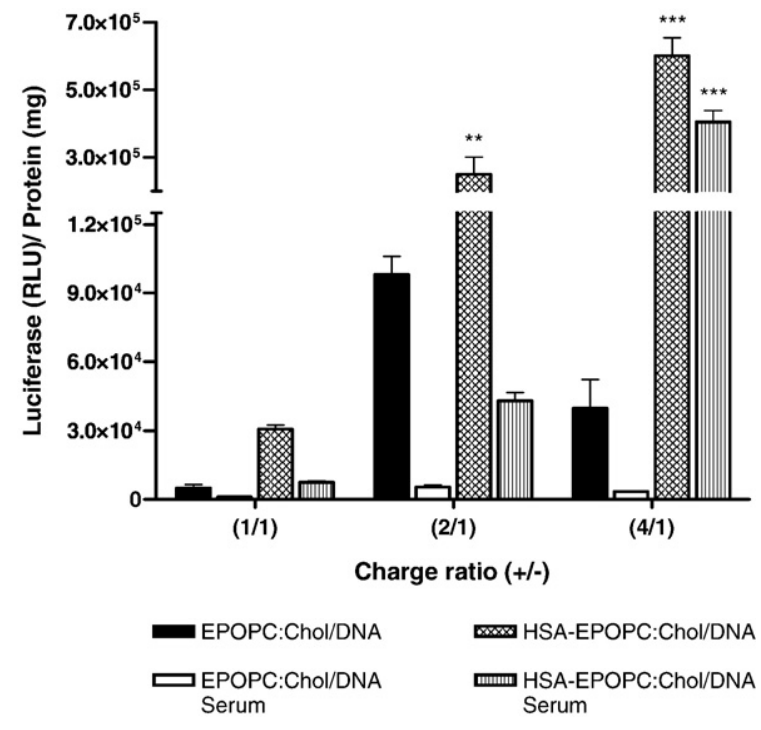

Fig. 2. Effect of albumin association to EPOPC:Chol/DNA lipoplexes on luciferase gene expression in TSA cells. Lipoplexes, prepared with or without HSA, were incubated with cells in the presence or absence of $10 \%$ serum. Levels of luciferase gene expression are presented as RLU of luciferase per mg of total cell protein (mean \pm S.D. obtained from triplicates), and are representative of at least three independent experiments. Asterisks $(* * p<0.01, * * * p<0.001)$ correspond to data from cells treated with HSA-associated lipoplexes which significantly differ from those obtained with cells treated with complexes prepared at the same charge ratio but without HSA.

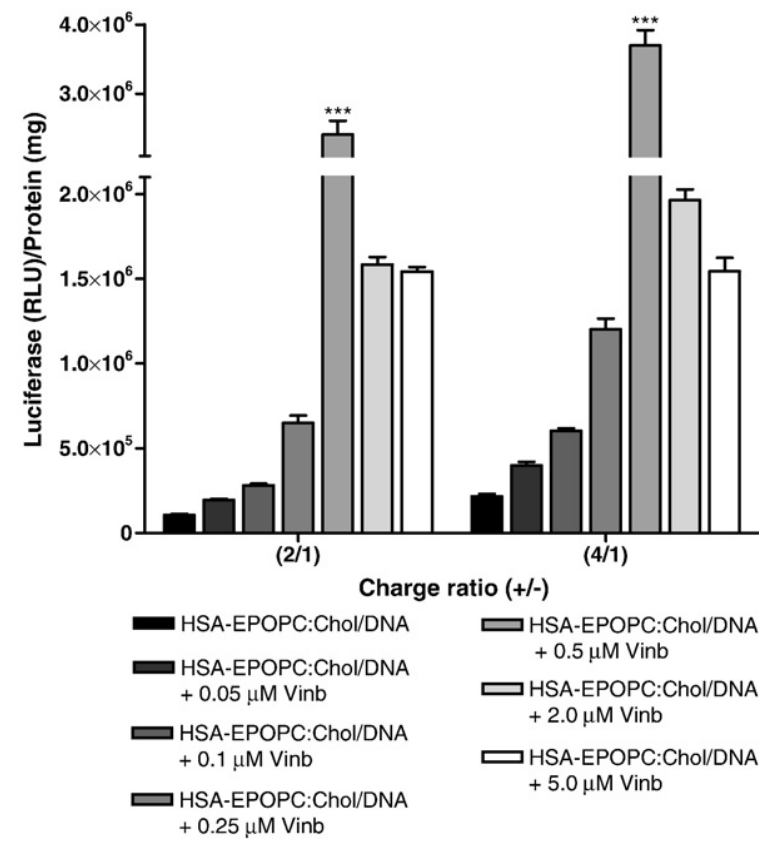

Fig. 3. Effect of vinblastine concentration on the transfection activity mediated by HSA-EPOPC:Chol/DNA lipoplexes in TSA cells. Cells were simultaneous incubated with lipoplexes and vinblastine $(0.05 ; 0.1 ; 0.25 ; 0.5 ; 2$ or $5 \mu \mathrm{M})$. Levels of luciferase gene expression are presented as RLU of luciferase per mg of total cell protein (mean \pm S.D. obtained from triplicates), and are representative of at least three independent experiments. Asterisks $\left({ }^{* * *} p<0.001\right)$, for the two best conditions, indicate values that significantly differ from those measured for all the other vinblastine concentrations at the same charge ratios. 

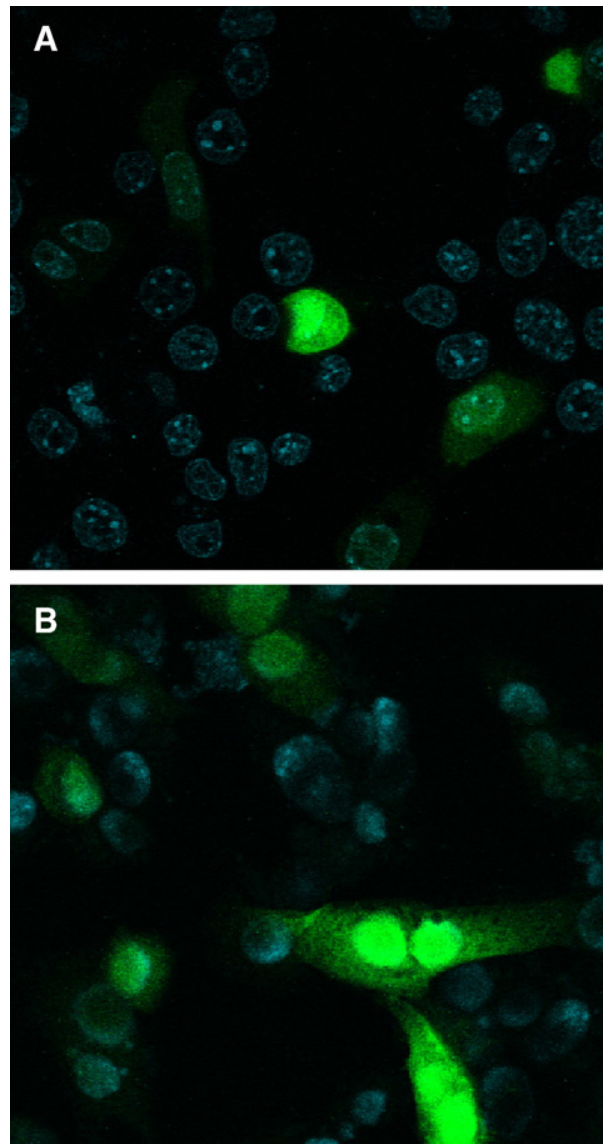

Fig. 4. Effect of vinblastine on gene delivery mediated by HSA-EPOPC:Chol/ DNA lipoplexes in TSA cells. Representative confocal microscopy images (original magnification: $\times 630$ ) of gene delivery mediated by (A) HSA-EPOPC: Chol/DNA (+/-) (4/1) lipoplexes and (B) HSA-EPOPC:Chol/DNA (+/-) (4/1) lipoplexes $+0.50 \mu \mathrm{M}$ vinblastine. TSA cells with Hoechst-labelled nuclei (blue) were transfected with lipoplexes containing the GFP plasmid.

activity, even in the presence of serum [10,11]. Therefore, in the present work, we tested this strategy in TSA cells for the two best liposome formulations, EPOPC:Chol and DOTAP:Chol, complexed with pCMVluc at the $(1 / 1),(2 / 1)$ and $(4 / 1)(+/-)$ charge ratios, in the presence or absence of serum. Fig. 2 illustrates the effect of albumin association to EPOPC:Chol/ DNA complexes on their biological activity. As can be observed, a great enhancement of transgene expression occurred particularly at the $2 / 1$ and $4 / 1(+/-)$ charge ratios $(p<0.01$ and $p<0.001$, respectively). As also shown in Fig. 2, when transfection experiments were performed in the presence of serum, a significant reduction in transgene expression was observed for plain lipoplexes, at all the charge ratios tested. However, resistance to the inhibitory effect of serum on biological activity was achieved for HSA-EPOPC:Chol/DNA lipoplexes prepared at the $(4 / 1)(+/-)$ charge ratio $(p<0.001)$.

The association of HSA to DOTAP:Chol/DNA lipoplexes also resulted in an enhancement of the transfection activity (data not shown), although to a lesser extent than that obtained for EPOPC:Chol/DNA lipoplexes. For this reason, the HSAEPOPC:Chol/DNA lipoplexes were selected for further studies.

\subsection{Effect of vinblastine on transfection activity of HSA-} EPOPC:Chol/DNA lipoplexes

Since the main goal of this work was to evaluate tumoral cell death resulting from the combination of vinblastine and nonviral HSV-tk/GCV gene therapy, we investigated whether this anti-cancer drug would have any effect as an enhancer of transfection activity mediated by lipoplexes. For this purpose, we addressed the effect of different concentrations of vinblastine on luciferase gene expression in TSA cells, mediated by HSA-EPOPC:Chol/DNA complexes prepared at (2/1) and (4/1) $(+/-)$ charge ratios. As can be observed in Fig. 3 , the presence of the chemotherapeutic agent resulted in an increase of the biological activity of the complexes. This enhancing effect was concentration-dependent, being maximal at $0.5 \mu \mathrm{M}$ vinblastine $(p<0.001)$, for both charge ratios (Fig. 3). Remarkably, at this concentration, vinblastine induced a 10 -fold increase in the transfection activity, even in the presence of serum (data not shown).

For all the vinblastine concentrations tested, the 4/1 lipid/ DNA $(+/-)$ charge ratio allowed greater levels of transgene expression than the $2 / 1(+/-)$ charge ratio, and therefore, HSAEPOPC:Chol/DNA (+/-) (4/1) lipoplexes were selected for further studies, namely those involving the delivery of the therapeutic gene.

The effect of vinblastine on transfection mediated by HSAEPOPC:Chol/DNA (+/-) (4/1) lipoplexes was also examined in TSA cells by confocal microscopy, in terms of the levels and

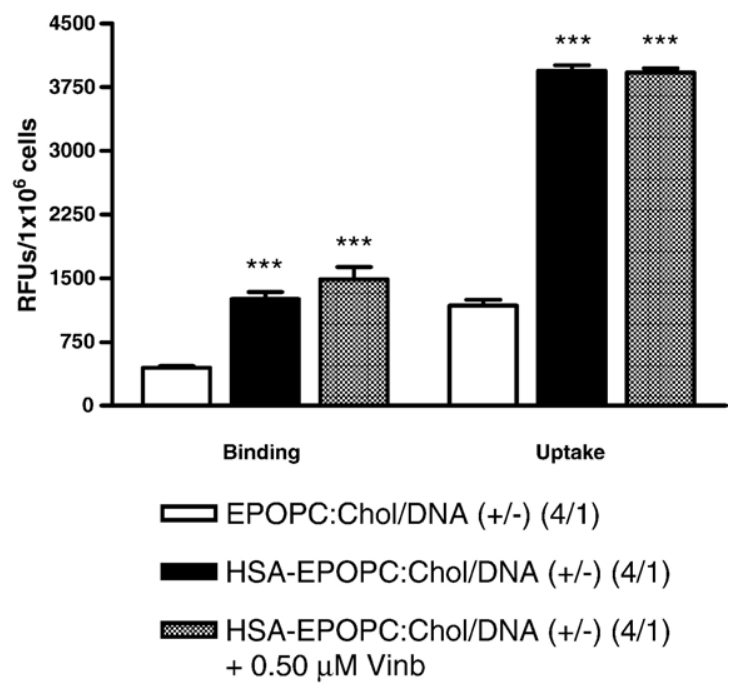

Fig. 5. Effect of vinblastine on the extent of binding and uptake of EPOPC:Chol/ DNA and HSA-EPOPC:Chol/DNA lipoplexes in TSA cells. Liposomes, labelled with rhodamine-PE, were associated or not to HSA before complexation with DNA. Cells were incubated for $2 \mathrm{~h}$ with the lipoplexes at $4{ }^{\circ} \mathrm{C}$ (binding) or $37^{\circ} \mathrm{C}$ (uptake), in the presence or absence of $0.5 \mu \mathrm{M}$ vinblastine (Vinb). The data are expressed as RFU per $10^{6}$ cells (mean \pm S.D. obtained from triplicates), and are representative of three independent experiments. Asterisks $\left({ }^{* * *} p<0.001\right)$ correspond to data from cells treated with HSA-associated lipoplexes which significantly differ from those obtained with cells treated with complexes prepared without HSA. 
extent of GFP gene expression. As illustrated in Fig. 4, the percentage of GFP-expressing cells and the intensity of GFP fluorescence were higher in the presence of $0.5 \mu \mathrm{M}$ of vinblatine. This observation is in agreement with the high levels of luciferase gene expression obtained in the presence of the same vinblastine concentration (Fig. 3).

\subsection{Binding and uptake of lipoplexes}

To investigate whether the enhancing effect of vinblastine on the biological activity of the complexes was due to an increase of binding and/or internalization of lipoplexes, TSA cells were incubated with rhodamine-PE-labeled EPOPC:Chol/DNA $(+/-)$

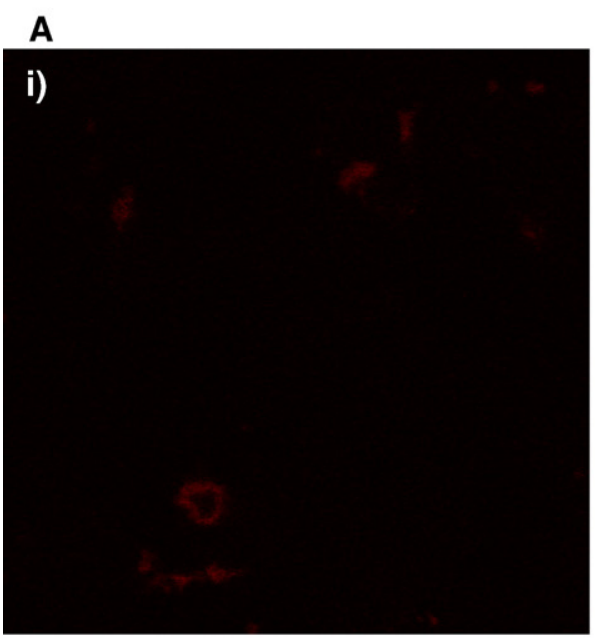

B
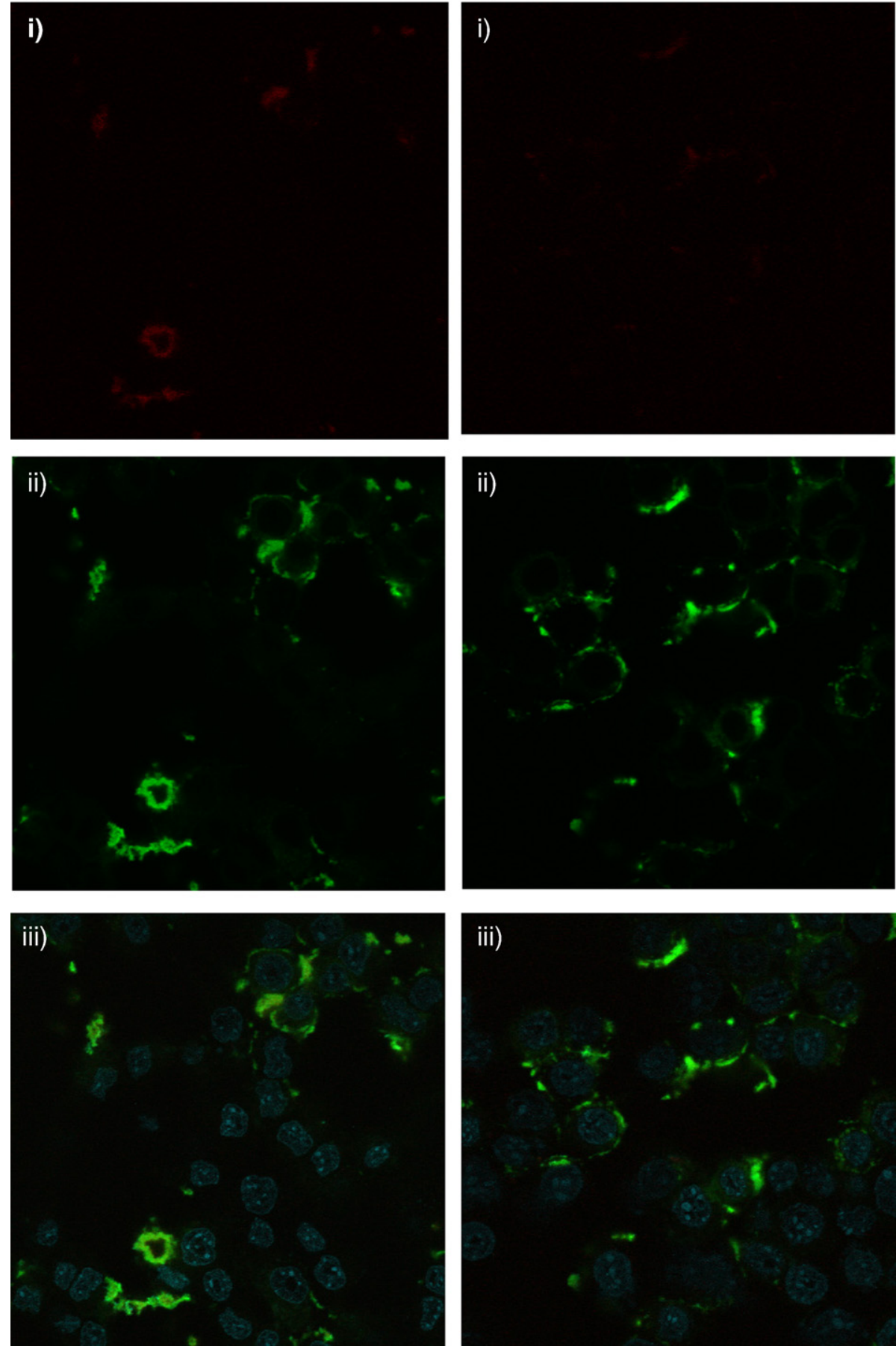

Fig. 6. Effect of vinblastine on the intracellular distribution of HSA-EPOPC:Chol/DNA lipoplexes. Representative confocal microscopy images (original magnification: $\times 630)$ of intracellular distribution, in TSA cells, of (A) HSA-EPOPC:Chol/DNA (+/-) (4/1) lipoplexes and (B) HSA-EPOPC:Chol/DNA (+/-) $(4 / 1)$ lipoplexes $+0.5 \mu \mathrm{M}$ vinblastine. (i) Endolysosomal pathway labelled with $200 \mathrm{M}$ of LysoTracker Red DND-99; (ii) Lipoplexes labelled with carboxyfluorescein; (iii) Co-localization of the lipoplexes and endolysosomal pathway in TSA cells with Hoechst-labelled nuclei. 
(4/1) or rhodamine-PE-labeled HSA-EPOPC:Chol/DNA (+/-) (4/1) lipoplexes at $4{ }^{\circ} \mathrm{C}$ (binding) and $37{ }^{\circ} \mathrm{C}$ (uptake), in the presence or absence of $0.5 \mu \mathrm{M}$ of vinblatine.

As can be observed in Fig. 5, association of albumin to lipoplexes resulted in a significant enhancement in the extent of binding $(p<0.001)$ and uptake $(p<0.001)$. On the other hand, the presence of vinblastine did not induce any significant change in the binding and uptake levels of HSA-EPOPC:Chol/ DNA (+/-) (4/1) lipoplexes (Fig. 5).

\subsection{Intracellular distribution of HSA-EPOPC:Chol/DNA lipoplexes}

We also examined the influence of vinblastine on the intracellular distribution of HSA-EPOPC:Chol/DNA (+/-) (4/1) lipoplexes by confocal microscopy. The endolysosomal pathway of TSA cells was labelled with LysoTracker Red, which labels acidic compartments in live cells, while the intracellular localization of the lipoplexes was visualized by incorporating carboxyfluorescein-PE into the liposomal membrane.

As shown in Fig. 6, in the presence of vinblastine HSAlipoplexes accumulated in a lower extent in the endolysosomal pathway, being more concentrated near the nuclei. These observations suggest that vinblastine most likely facilitates delivery of the complexes from the endocytotic pathway. On the other hand, this drug did not interfere with the cellular uptake of HSA-associated lipoplexes (identical intensity levels of GFP fluorescence in Fig. 6 A and B), which is in agreement with the results shown in Fig. 5.

\subsection{Antitumoral activity}

The main goal of this study was to evaluate the therapeutic potential resulting from the combination of two antitumoral strategies: HSV-Tk/GCV suicide gene therapy and conventional chemotherapy. For this purpose, TSA cells were treated with vinblastine (chemotherapeutic agent) or transfected with HSAEPOPC:Chol/DNA (+/-) (4/1) lipoplexes carrying the pCMVtk plasmid, in the presence (combined therapy) or absence of $0.5 \mu \mathrm{M}$ vinblastine, and then incubated for five days with different concentrations of valganciclovir (VGCV).

As shown in Fig. 7, the cytotoxic effect induced by the combined or HSV-tk/GCV gene therapy approaches is dependent both on VGCV concentration and incubation time. The most effective concentration was $400 \mu \mathrm{M} \mathrm{VGCV}$, no significant differences being observed for the extent of cytotoxicity induced by 100 or $200 \mu \mathrm{M}$ VGCV. For all tested strategies, the highest reduction in cell viability was obtained at the fifth day of treatment.

In the absence of vinblastine $54 \%$ of cell death $(p<0.001)$ was achieved after transfection followed by five days of treatment with $400 \mu \mathrm{M}$ VGGV. On the other hand, for non-transfected cells that were treated with $0.5 \mu \mathrm{M}$ vinblastine, only $10 \%$ reduction of cell viability was obtained. However, for cells that were transfected in the presence of $0.5 \mu \mathrm{M}$ vinblastine (combined therapy), five days of treatment with $400 \mu \mathrm{M}$ VGGV resulted in an almost total cell death ( $98 \%$ of cytotoxicity),
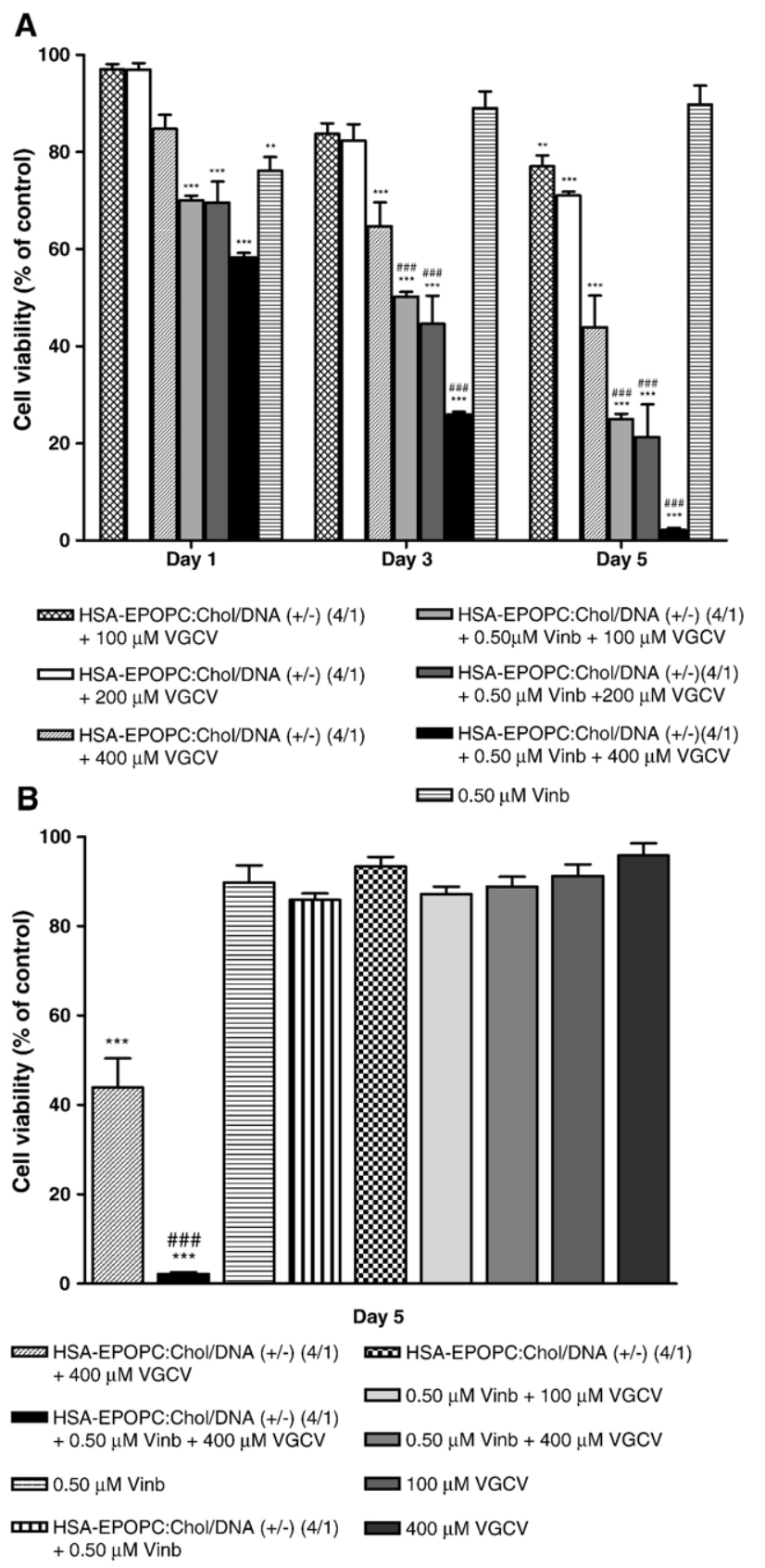

Fig. 7. Effect of valganciclovir concentration and incubation time on cell viability. TSA cells were incubated with HSA-EPOPC:Chol/DNA (+/-) (4/1) lipoplexes, containing $1 \mu \mathrm{g}$ of pCMVtk plamid, or HBS (non-transfected cells), in the presence or absence of $0.5 \mu \mathrm{M}$ vinblastine. Following $24 \mathrm{~h}$, the medium was replaced with DMEM-HG (control cells and untreated cells with VGCV) or with DMEM-HG containing different concentrations of VGCV (100, 200 or $400 \mu \mathrm{M})$. Medium was replaced daily and cell viability measured by Alamar Blue assay. The data are expressed as the percentage of the untreated control cells (mean \pm standard deviation obtained from triplicates) and are representative of at least four independent experiments. The results illustrated in (B) correspond to the cell viability measured at the fifth day of treatment. Asterisks $\left({ }^{* *} p<0.01,{ }^{* * *} p<0.001\right)$ indicate values that significantly differ from those measured for non-treated cells (control). Cardinals (\#\#\#<0.001) correspond to data from cells treated with the combined therapy which significantly differ from those obtained with cells treated with each strategy alone. 
showing a significant synergistic effect $(p<0.001)$ from the combination of these two therapeutic strategies. This synergistic enhancement of cytotoxicity was observed for the three VGCV tested concentrations (Fig. 7A). As illustrated in Fig. 7B (cell viability for the fifth day of treatment), VGCV was non-toxic to non-transfected cells, either per se or in the presence of $0.5 \mu \mathrm{M}$ vinblastine, and no significant toxicity was induced upon transfection of cells in the absence of VGCV treatment. Under these control conditions no significant toxicity was observed for days 1 and 3 of treatment (data not shown). Therefore, the reduction in cell viability observed in transfected cells after treatment with VGCV is not due to VGCV itself or to the transfection protocol.

\section{Discussion}

Due to their simplicity, ease of large-scale production and lack of specific immune response, cationic liposome/DNA complexes constitute a promising tool for delivery of therapeutic genes in genetic and acquired human diseases. However, the transgene expression mediated by these non-viral carriers is inefficient, specially in vivo, being highly influenced by their physico-chemical properties $[1-4,29]$. In this context, the identification, understanding and the modulation of these parameters are crucial to develop new efficient non-viral gene delivery strategies.

As shown in Fig. 1, among the different liposome formulations tested, EPOPC:Chol and DOTAP:Chol were those resulting in complexes with the highest levels of transfection $(p<0.001$ and $p<0.01$, respectively). In agreement with other reports, these results show that the composition of the cationic liposomes has a great importance for the biological activity of the complexes $[5,29]$. On the other hand, the charge ratio of lipid/DNA complexes is also a crucial factor in the lipofection process, since it determines the surface charge and the mean diameter of the complexes $[5,6]$. In this regard, for the most efficient lipoplexes (EPOPC:Chol/DNA and DOTAP:Chol/ DNA), the highest transgene expression obtained for the complexes with positive zeta potential, (2/1) and (4/1) (+/-) lipoplexes, can be explained by a greater extent of interaction with the negatively charged cell membrane [29]. Although, the (4/1) $(+/-)$ lipoplexes exhibit a more positive surface charge than those prepared at a $(2 / 1)(+/-)$ charge ratio, they revealed a lower biological activity than $(2 / 1)(+/-)$ lipoplexes. This observation may be explained by the smaller sizes of the positively charged (4/1) (+/-) complexes as compared to the $(2 / 1)(+/-)$ complexes and by the extensive condensation of genetic material, which can limit the intracellular delivery of DNA, as shown in our previous study [29].

Several approaches have been tested aiming at improving the capacity of lipoplexes to mediate intracellular delivery of genetic material. In agreement with our previous findings [10,11], the results obtained in this work (Fig. 2) revealed that association of albumin to lipoplexes is an efficient approach, resulting in a great enhancement of transgene expression, although depending on the lipid composition and cationic lipid/DNA (+/-) charge ratio of lipoplexes. For the two tested liposome formulations, EPOPC:Chol and DOTAP:Chol, the most pronounced enhancing effect mediated by HSA was observed for EPOPC: Chol/DNA complexes, particularly at the $(4 / 1)(+/-)$ charge ratio $(p<0.001)$, even in the presence of serum $(p<0.001)$. This enhancing effect of albumin is most probably dependent on the lipid nature of the liposomes, which may explain the high transfection activity of HSA-EPOPC:Chol/DNA lipoplexes. The lipid nature could not only influence the capacity of HSA to mediate the increase of biological activity, but could also determine the final structure/properties of the complexes containing HSA and consequently their transfection capacity $[11,30]$.

As indicated by the results from binding and uptake studies (Fig. 5), association of HSA to EPOPC:Chol/DNA (4/1) (+/-) lipoplexes resulted in a 3 -fold enhancement $(p<0.001)$ of binding and cellular uptake. However, the biological activity studies (Fig. 2) show that association of HSA to EPOPC:Chol/ DNA (4/1) (+/-) lipoplexes resulted in an increase of 15 -fold $(p<0.001)$ in the levels of transgene expression. Since the increase of transgene expression achieved upon HSA association was nearly 5 -fold higher than that for the increase in the uptake of HSA-associated lipoplexes, it seems reasonable to assume that HSA may act at two different stages of the endocytotic pathway: by promoting binding/endocytosis of the lipoplexes and by facilitating escape of DNA from the endosome [31-33].

The intracellular traffic of cationic liposome/DNA complexes has been the subject of several studies aiming at identifying the cellular barriers that limit efficient gene delivery to target cells. To this regard, the endolysosomal entrapment of lipoplexes has been identified as one of the major limiting factors of the transfection efficiency [5-9].

In this work, we tested the potential of the microtubuledepolymerizing agent vinblastine to enhance transgene expression mediated by HSA-EPOPC:Chol/DNA lipoplexes in TSA cells, probably by decreasing the intracellular traffic of lipoplexes to lysosomes and/or activating the transcription factor $\mathrm{NF}-\mathrm{kb}$. In face of the wide clinical application of vinblastine as an anticancer agent, gene delivery mediated by lipoplexes in the presence of this drug may be an efficient antitumoral approach due to its dual effect: enhancement of transgene expression and chemotherapeutic activity. The results illustrated in Figs. 3 and 4 reveal that vinblastine significantly $(p<0.001)$ improved transgene (luciferase and GFP) expression in TSA cells, when used at a concentration of $0.5 \mu \mathrm{M}$. The confocal microscopy images of GFP gene expression (Fig. 4) show that such enhancement translated both into higher levels of gene expression and larger percentage of transfected cells.

Aiming at gaining insights into the mechanisms involved in the vinblastine effect, binding, uptake and intracellular distribution studies were performed with HSA-EPOPC:Chol/ DNA (+/-) (4/1) lipoplexes in the presence of $0.5 \mu \mathrm{M}$ vinblastine. As shown in Figs. 5 and 6, the extent of internalized lipoplexes is similar in the presence and absence of vinblastine, thus the potentiation of the transfection activity mediated by this drug was not the result of the enhancement of lipoplex uptake. This observation is consistent with the reported role of actin rather than that of microtubules as the 
element responsible for the initial binding and uptake by endocytosis $[34,35]$. Vinblastine probably enhances transgene expression by decreasing the intracellular traffic of lipoplexes to lysosomes and/or by activating the nuclear factor NF- $\kappa \mathrm{B}$ and consequently transgene transcription [23-27]. The lower accumulation of HSA-EPOPC:Chol/DNA lipoplexes observed in the endolysosomal pathway in the presence of vinblastine (Fig. 6) suggests that the depolymerization of the microtubules results in fast delivery (escape) of the lipoplexes from the endocytotic pathway. The small difference observed for the intracellular distribution of lipoplexes in the presence and absence of vinblastine can result from the association of HSA to lipoplexes, since HSA can also promote their escape from the endocytotic pathway, thus partially masking the effect of vinblastine.

The effect of vinblastine on the antitumoral activity of the HSV-tk/GCV "suicide" gene therapy mediated by HSA-associated lipoplexes was evaluated in TSA cells and compared with that observed upon application of the chemotherapeutic agent and the gene therapy strategy (HSV-tk/GCV), separately. The results illustrated in Fig. 7 reveal that the highest antitumoral activity was observed for the combined therapy (HSV-tk/VGCV gene therapy + vinblastine), at the fifth day of treatment with $400 \mu \mathrm{M} \mathrm{VGCV} \mathrm{(} \mathrm{100 \%} \mathrm{of} \mathrm{cytotoxicity).} \mathrm{The}$ huge difference $(p<0.001)$ observed between the cytotoxicity achieved by application of the combined therapy $(\sim 100 \%)$ and that obtained for each strategy per se $(54 \%$ for the gene therapy strategy and $10 \%$ for the treatment with vinblastine) clearly shows the occurrence of a synergistic effect. We have also observed (data not shown) that the application of vinblastine alone requires a concentration of $250 \mu \mathrm{M}$ (500 times higher than that used in the combined therapy) to obtain the same cytotoxic effect as that achieved with the combined therapy. Such synergistic effect may be the result of the dual effect of vinblastine, by enhancing HSV-tk gene expression, with a subsequent increase in the amount of the toxic metabolite (GCV-TP), and arresting the cell cycle at the mitosis level.

\section{Conclusion}

Overall, the results presented in this manuscript show that the combination of vinblastine with the HSV-Tk/GCV gene therapy, mediated by HSA-associated lipoplexes, results in a significant synergistic effect. Such effect allows the use of a much lower dose of the drug to achieve a therapeutic activity and consequently to reduce side effects, thus highlighting the potential of this combined strategy for future clinical antitumoral applications.

\section{Acknowledgements}

H. Faneca is a recipient of a fellowship from the Portuguese Foundation for Science and Technology (SFRH/BPD/20494/ 2004/5ISA). This work was financed by grants from the Portuguese Foundation for Science and Technology (POCTI/ CVT/44854/2002 and PTDC/BIO/65627/2006).

\section{References}

[1] V.A. Rakhmanova, E.V. Pozharski, R.C. MacDonald, Mechanisms of lipoplex formation: dependence of the biological properties of transfection complexes on formulation procedures, J. Membr. Biol. 200 (2004) 35-45.

[2] M.C. Pedroso de Lima, S. Simões, P. Pires, H. Faneca, N. Düzgünes, Cationic lipid-DNA complexes in gene delivery: from biophysics to biological applications, Adv. Drug Deliv. Rev. 47 (2001) 277-294.

[3] F. Sakurai, R. Inoue, Y. Nishino, A. Okuda, O. Matsumoto, T. Taga, F. Yamashita, Y. Takakura, M. Hashida, Effect of DNA/liposome mixing ratio on the physicochemical characteristics, cellular uptake and intracellular trafficking of plasmid DNA/cationic liposome complexes and subsequent gene expression, J. Control. Release 66 (2000) 255-269.

[4] P.C. Ross, S.W. Hui, Lipoplex size is a major determinant of in vitro lipofection efficiency, Gene Ther. 6 (1999) 651-659.

[5] L. Wasungu, D. Hoekstra, Cationic lipids, lipoplexes and intracellular delivery of genes, J. Control. Release 116 (2006) 255-264.

[6] I.A. Khalil, K. Kogure, H. Akita, H. Harashima, Uptake pathways and subsequent intracellular trafficking in nonviral gene delivery, Pharmacol. Rev. 58 (2006) 32-45. S.

[7] Simões, A. Filipe, H. Faneca, M. Mano, N. Penacho, N. Düzgünes, M.C. Pedroso de Lima, Cationic liposomes for gene delivery, Expert Opin. Drug Deliv. 2 (2005) 237-254.

[8] D. Lechardeur, G.L. Lukacs, Intracellular barriers to non-viral gene transfer, Curr. Gene Ther. 2 (2002) 183-194.

[9] V. Escriou, C. Ciolina, A. Helbling-Leclerc, P. Wils, D. Scherman, Cationic lipid-mediated gene transfer: analysis of cellular uptake and nuclear import of plasmid DNA, Cell Biol. Toxicol. 14 (1998) 95-104.

[10] H. Faneca, A.S. Cabrita, S. Simões, M.C. Pedroso de Lima, Evaluation of the antitumoral effect mediated by IL-12 and HSV-tk genes when delivered by a novel lipid-based system, Biochim. Biophys. Acta 1768 (2007) 1093-1102.

[11] H. Faneca, S. Simões, M.C. Pedroso de Lima, Association of albumin or protamine to lipoplexes: enhancement of transfection and resistance to serum, J. Gene Med. 6 (2004) 681-692.

[12] R.M. Hughes, Strategies for cancer gene therapy, J. Surg. Oncol. 85 (2004) $28-35$.

[13] M. Cavazzana-Calvo, A. Thrasher, F. Mavilio, The future of gene therapy, Nature 427 (2004) 779-781.

[14] A. El-Aneed, An overview of current delivery systems in cancer gene therapy, J. Control. Release 94 (2004) 1-14.

[15] K. Yazawa, W.E. Fisher, F.C. Brunicardi, Current progress in suicide gene therapy for cancer, World J. Surg. 26 (2002) 783-789.

[16] M. Aghi, F. Hochberg, X.O. Breakefield, Prodrug activation enzymes in cancer gene therapy, J. Gene Med. 2 (2000) 148-164.

[17] A. Ketola, A.M. Maatta, T. Pasanen, K. Tulimaki, J. Wahlfors, Osteosarcoma and chondrosarcoma as targets for virus vectors and Herpes simplex virus thymidine kinase/ganciclovir gene therapy, Int. J. Mol. Med. 13 (2004) 705-710.

[18] J. Wang, X.X. Lu, D.Z. Chen, S.F. Li, S.L. Zhang, Herpes simplex virus thymidine kinase and ganciclovir suicide gene therapy for human pancreatic cancer, World J. Gastroenterol. 10 (2004) 400-403.

[19] A.M. Maatta, A. Tenhunen, T. Pasanen, O. Merilainen, R. Pellinen, K. Makinen, E. Alhava, J. Wahlfors, Non-small cell lung cancer as a target disease for Herpes simplex type 1 thymidine kinase-ganciclovir gene therapy, Int. J. Oncol. 24 (2004) 943-949.

[20] F.W. Floeth, N. Shand, H. Bojar, H.B. Prisack, J. Felsberg, E. NeuenJacob, A. Aulich, K.J. Burger, W.J. Bock, F. Weber, Local inflammation and devascularization: in vivo mechanisms of the "bystander effect" in VPC-mediated HSV-Tk/GCV gene therapy for human malignant glioma, Cancer Gene Ther. 8 (2001) 843-851.

[21] R. Ramesh, A.J. Marrogi, S.M. Freeman, Tumor killing using the HSV-tk suicide gene, Gene Ther. Mol. Biol. 1 (1998) 253-263.

[22] R. Ramesh, A.J. Marrogi, A. Munshi, C.N. Abboud, S.M. Freeman, In vivo analysis of the "bystander effect": a cytokine cascade, Exp. Hematol. 24 (1996) 829-838.

[23] Y. Huang, Y. Fang, J. Wu, J.M. Dziadyk, X. Zhu, M. Sui, W. Fan, Regulation of Vinca alkaloid-induced apoptosis by NF-KB/IKB pathway in human tumor cells, Mol. Cancer Ther. 3 (3) (2003) 271-277. 
[24] L. Wang, R.C. MacDonald, Effects of microtubule-depolymerizing agents on the transfection of cultured vascular smooth muscle cells: enhanced expression with free drug and especially with drug-gene lipoplexes, Mol. Ther. 9 (5) (2004) 729-737.

[25] S. Hasegawa, N. Hirashima, M. Nakanishi, Microtubule involvement in the intracellular dynamics for gene transfection mediated by cationic liposomes, Gene Ther. 8 (2001) 1669-1673.

[26] N.R. Chowdhury, R.M. Hays, V.R. Bommineni, N. Franki, J.R. Chowdhury, C.H. Wu, G.Y. Wu, Microtubular disruption prolongs the expression of human bilirubin-uridinediphosphoglucuronate-glucuronosyltransferase-1 gene transferred into gunn rat livers, J. Biol. Chem. 271 (4) (1996) 2341-2346.

[27] C. Rosette, M. Karin, Cytoskeletal control of gene expression: depolymerization of microtubules activates NF-kB, J. Cell Biol. 128 (6) (1995) $1111-1119$.

[28] K. Konopka, E. Pretzer, P.L. Felgner, N. Düzgünes, Human immunodeficiency virus type-1 (HIV-1) infection increases the sensitivity of macrophages and THP-1 cells to cytotoxicity by cationic liposomes, Biochim. Biophys. Acta 1312 (1996) 186-196.
[29] H. Faneca, S. Simões, M.C. Pedroso de Lima, Evaluation of lipid-based reagents to mediate intracellular gene delivery, Biochim. Biophys. Acta 1567 (2002) 23-33.

[30] S. Schenkman, P.S. Araujo, R. Dijkman, F.H. Quina, H. Chaimovich, Effects of temperature and lipid composition on the serum albumininduced aggregation and fusion of small unilamellar vesicles, Biochim. Biophys. Acta 649 (1981) 633-647.

[31] K.K. Sorensen, J. Melkko, B. Smedsrod, Scavenger-receptor-mediated endocytosis in endocardial endothelial cells of Atlantic cod Gadus morhua, J. Exp. Biol. 201 (1998) 1707-1718.

[32] R. Nunes, C.L. Kiang, D. Sorrentino, P.D. Berk, "Albumin-receptor" uptake kinetics do not require an intact lobular architecture and are not specific for albumin, J. Hepatol. 7 (1988) 293-304.

[33] J. Wilschut, D. Hoekstra, Membrane fusion: lipid vesicles as a model system, Chem. Phys. Lipids 40 (1986) 145-166.

[34] S. Conner, S. Schmid, Regulated portals of entry into the cell, Nature 422 (6927) (2003) 37-44.

[35] A. Durrbach, D. Louvard, E. Coudrier, Actin filaments facilitate two steps of endocytosis, J. Cell Sci. 109 (1996) 457-465. 